\title{
Development of Advanced Tokamak Operation Scenarios in JT-60U and JT-60SA
}

\author{
Takahiro SUZUKI and the JT-60 Team \\ Japan Atomic Energy Agency, 801-1, Mukoyama, Naka, Ibaraki 311-0193, Japan
}

(Received 7 December 2009 / Accepted 9 March 2010)

\begin{abstract}
Integrated high performance was achieved in a wall-stabilized reversed shear plasma, and the high values of $H_{\mathrm{H} 98(\mathrm{y}, 2)}=1.7, \beta_{\mathrm{N}}=2.7, f_{\mathrm{GW}}=0.87, f_{\mathrm{BS}}=0.9$, and $f_{\mathrm{NI}}=0.95$ were simultaneously realized at the ITER/DEMOrelevant value of $q_{95}=5.3$. The $\beta_{\mathrm{N}}$ value achieved was above the no-wall $\beta_{\mathrm{N}}$ limit of 1.9 and close to the ideal-wall $\beta_{\mathrm{N}}$ limit of 2.9 in that discharge. The $\beta_{\mathrm{N}}$ was limited by the appearance of the Resistive Wall Mode (RWM) when the toroidal rotation velocity at the $q=3$ surface decreased to below the critical velocity. Another discharge realized a fully relaxed current profile at the high value of $f_{\mathrm{BS}}=0.5$ in a fully non-inductive Current Drive (CD) condition at $q_{95}=5.8$ through use of a lower-hybrid CD and Neutral Beam (NB) CDs with bootstrap current. Integration of operation scenarios to ensure steady-state high performance is a major mission with the JT-60SA. A DEMO-equivalent value of $\beta_{\mathrm{N}}=4.3$ is being aimed at utilizing highly shaped configurations, closely-placed conducting walls, low ripple rates, and RWM control coils. Steady-state $q$ profiles can be maintained using an off-axis CD resulting from a negative-ion based NB and bootstrap current for 60-100 s. An operation scenario that supports building up the $I_{\mathrm{p}}$ with less consumption of the magnetic flux is a key issue in the realization of a compact DEMO SlimCS. Fully non-inductive buildup of $I_{\mathrm{p}}$ and recharging of the Ohmic coil current were demonstrated utilizing the JT-60U. Development of an overall operation scenario from the buildup of the $I_{\mathrm{p}}$ to steady sustainment of a high performance plasma is an important challenge with the JT-60SA.
\end{abstract}

(C) 2010 The Japan Society of Plasma Science and Nuclear Fusion Research

Keywords: advanced tokamak, steady-state operation scenario, wall-stabilization, resistive wall mode, non-inductive current drive, bootstrap current, JT-60U, JT-60SA, ITER, DEMO

DOI: $10.1585 /$ pfr.5.S2008

\section{Introduction}

The JT-60U program [1] has taken place in order to contribute to ITER and DEMO through providing physics basis and Advanced Tokamak (AT) operation scenarios. In August 2008 JT-60U successfully completed operation, and the construction of the superconducting tokamak JT60SA is now in progress. The JT-60SA supports ITER and will contribute to early realization of fusion energy in DEMO by complementing ITER. AT operation scenarios have been introduced in contrast to the conventional inductive $\mathrm{H}$-mode tokamak operation scenario, and are currently being intensively studied worldwide.

A tokamak plasma is confined by the rotational transform produced by the plasma current of $I_{\mathrm{p}}$. In a conventional tokamak, the $I_{\mathrm{p}}$ is mainly driven inductively by temporal changes in the magnetic flux $(\mathrm{d} \Psi / \mathrm{d} t)$, where the magnetic flux $\Psi$ is provided by $\mathrm{Ohmic}(\mathrm{OH})$ coil current $\left(I_{\mathrm{OH}} \propto \Psi\right)$. The $I_{\mathrm{OH}}$ is limited however, and hence inductive operations in the conventional tokamak have not supported steady-state operation. The AT operation concept involves the realization of a steady-state tokamak operation non-inductively that makes full use of the bootstrap current of $I_{\mathrm{BS}}$ which is driven by the pressure gradient in the torus system. This necessitates that the non-inductive

author'se-mail: suzuki.takahiro@jaea.go.jp current $I_{\mathrm{NI}}$, which is the sum of the bootstrap current and current driven by the external Current Drive (CD) system of $I_{\mathrm{CD}}$, must be equal to $I_{\mathrm{p}}$ in achieving steady-state operation. The external CD system requires large amounts of electricity and hence as large an $I_{\mathrm{BS}}$ as possible is necessary in ensuring the reactor is economical. In AT operations the fraction of bootstrap current to plasma current $f_{\mathrm{BS}} \equiv I_{\mathrm{BS}} / I_{\mathrm{p}}$ is a key figure of merit, along with the fraction of non-inductive current to plasma current $f_{\mathrm{NI}} \equiv I_{\mathrm{NI}} / I_{\mathrm{p}}$ in steady-state operation. Other figures of merit from the view point of the core plasma include the $H_{\mathrm{H} 98(\mathrm{y}, 2)}$ for the thermal energy confinement time with respect to ELMy Hmode scaling [2], $\beta_{\mathrm{N}}$ (normalized beta) for pressure or stability, $f_{\mathrm{GW}}$ for electron density with respect to the Greenwald density limit, and the $1 / q_{95} \propto I_{\mathrm{p}} / B_{\mathrm{t}}$ for the plasma current of given toroidal field, where $q_{95}$ is the safety factor $(q)$ at $95 \%$ of the magnetic surface close to the plasma edge. The 6 figures of merit described above for an ITER steady-state operation scenario with a Reversed magnetic Shear $(\mathrm{RS})$ [3] are $f_{\mathrm{BS}}=0.46, f_{\mathrm{NI}}=1$ (full-CD), $H_{\mathrm{H} 98(\mathrm{y}, 2)}=$ $1.6, \beta_{\mathrm{N}}=2.9, f_{\mathrm{GW}}=0.78$ and $1 / q_{95}=1 / 5.4$. However, for integrated high performance, larger figures of merit need to be simultaneously achieved. In steady-state operation, the integrated high performance must be sustained sufficiently long for at least the current profile to reach a steady- 
state profile as the confinement and stability of a tokamak is known to be strongly affected by the current profile of the plasma. A time scale for the current profile relaxation in the tokamak is $\tau_{\mathrm{R}}$ [4], which is of the order of $1 \mathrm{~s}$ and longer than the energy confinement time of the order of $0.1 \mathrm{~s}$ for a typical JT-60U plasma.

The current profile or safety factor profile of the tokamak will be briefly described here (refer to Figure 1). A minimum safety factor of $q_{\min }$ is typically about 1 with a conventional tokamak, being mainly due to the centrally peaked neo-classical electric conductivity and corresponding inductive current. In AT operations, the $q_{\text {min }}$ needs to be maintained at larger than 1.5 (or 2) to eliminate low$m \& n$-mode-resonant rational $q$ surfaces in avoiding any MHD activity, such as sawtooth and Neoclassical Tearing Mode (NTM), where $m$ and $n$ are the poloidal and toroidal mode numbers, respectively. Also in AT operations small or zero inductive current and large bootstrap current helps to broaden the current profile and thus raise the $q_{\min }$. With any increase in the off-axis bootstrap current and externally driven current the $q$ profile changes from a Weak magnetic Shear (WS) regime to an RS regime. This then makes exploring the optimum $q$ profile from the viewpoint of stable and steady sustainment of an integrated high performance plasma rather important when developing AT operation scenarios.

This paper presents the development of AT operation scenarios in the JT-60U and the prospects with JT-60SA in view of ITER and DEMO (such as SlimCS [5]). Section 2 then describes the integrated high performance achieved in a wall-stabilized RS plasma. Section 3 is allocated to the development of a full-CD high $f_{\mathrm{BS}}$ plasma with a fully relaxed current profile as these conditions are very much required in ensuring the stable steady-state operation of a tokamak reactor. Section 4 is dedicated to the AT operation scenarios being designed for JT-60SA and to be developed for JT-60SA in contributing to ITER and DEMO. A summary is provided in Section 5.

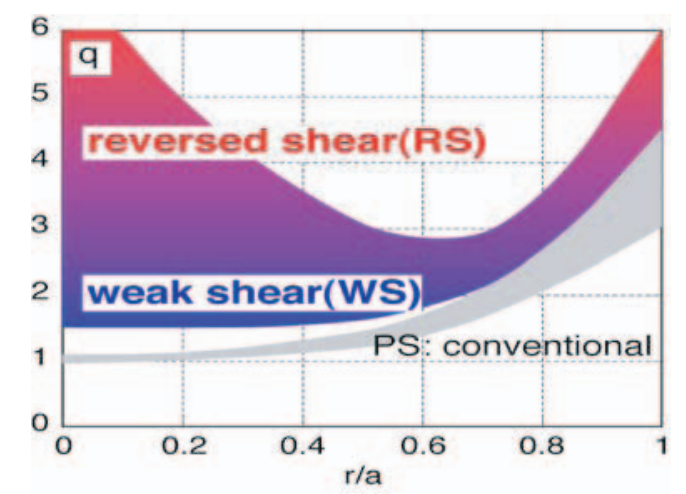

Fig. 1 Safety factor $(q)$ profile of AT operation scenarios with reversed magnetic shear regime and weak magnetic shear regime in contrast to conventional operation scenario with Positive Shear (PS).

\section{Development of Integrated High Performance Plasma in JT-60U}

In JT-60U, an RS operation scenario resulted in a steady-state current profile with the high values of $H_{\mathrm{H} 98(\mathrm{y}, 2)}$ $=1.9$ and $f_{\mathrm{BS}}=0.75$, and the $f_{\mathrm{NI}}=0.94$ (e43046) [6], however, the stability was low $\left(\beta_{\mathrm{N}}=1.7\right)$ and the $q_{95}$ still rather high (8.3). This scenario was developed for use with an inboard-shifted small volume plasma (refer to Figure 2) in avoiding a large toroidal field ripple region that exists in the outboard region of the torus and that causes a ripple loss in the heating NBs. Ferritic Steel Tiles (FSTs) were installed in order to reduce that ripple and enable wall-stabilization by allowing the plasma to be positioned close to the wall (vacuum vessel in JT-60U) [7].

That modification enabled an RS operation scenario to be developed in the outboard-shifted close-wall condition (refer to Figure 2) [8]. The ratio of wall radius $(d)$ to plasma minor radius $(a)$ decreased from $d / a \sim 1.5$ (e43046) to $\sim 1.3$ (e48246). Figure 3 provides the waveforms of the discharge with a flattop $I_{\mathrm{p}}=0.8 \mathrm{MA}, B_{\mathrm{t}}=2 \mathrm{~T}$ and $q_{95}=$ 5.3 (e48246). A high integrated performance plasma with a high $H_{\mathrm{H} 98(\mathrm{y}, 2)}=1.7, f_{\mathrm{GW}}=0.98, f_{\mathrm{BS}} \sim 0.92$ and $\beta_{\mathrm{N}} \sim$ 2.7 above the no-wall beta limit of $\sim 1.9$ was realized at an ITER/DEMO-relevant $q_{95} \sim 5.3$ in an RS regime through the wall-stabilization. Note that the very good confinement at $H_{\mathrm{H} 98(\mathrm{y}, 2)}=1.7$ was obtained at a high electron density close to the Greenwald density limit $\left(f_{\mathrm{GW}}=0.98\right)$. In this experiment, the non-inductive CD fraction (0.94) was slightly lower than unity. The performance of the plasma is detailed in Figure 4, using the 6 figures of merit described in the introduction. Note that all the figures of merit were normalized to those of the ITER steady-state RS operation scenario [3]. The figures of merit with regard to DEMO (SlimCS [5]) are also indicated. The figures of merit with the JT-60U RS discharge in Figure 3 almost cover those of

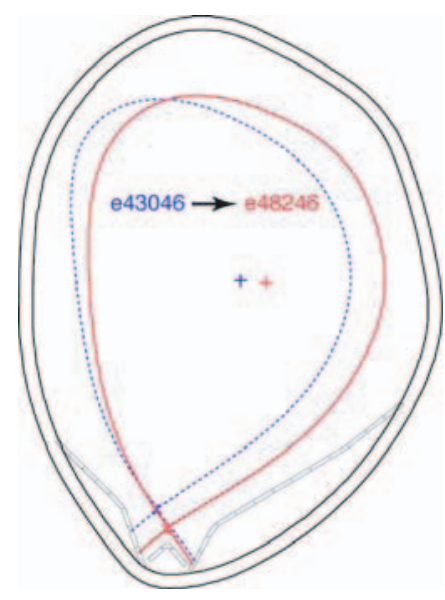

Fig. 2 Configuration of RS plasma in JT-60U before (dotted) and after (solid) installation of FSTs with respect to vacuum vessel (conducting wall). The ratio of wall radius (d) to plasma minor radius $(a)$ decreased from $d / a \sim 1.5$ to $\sim 1.3$. 

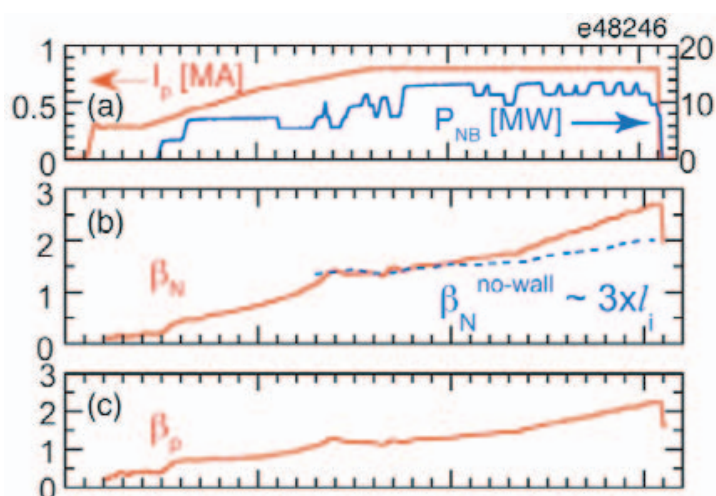

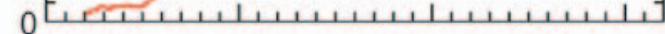
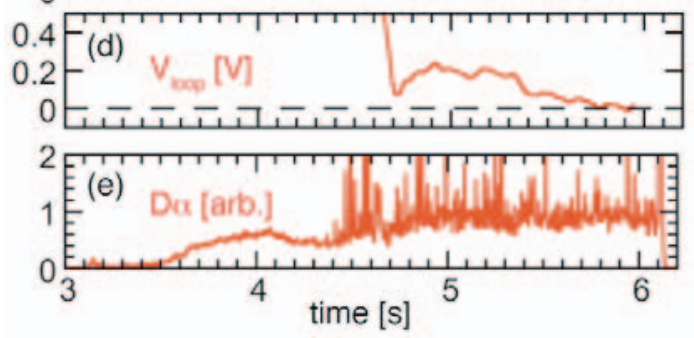

Fig. 3 Waveforms of wall-stabilized RS discharge in JT-60U: (a) plasma current $I_{\mathrm{p}}$ and NB heating power $P_{\mathrm{NB}}$, (b) normalized beta $\beta_{\mathrm{N}}$ with 3 times the internal inductance $l_{\mathrm{i}}$ as a measure of no-wall $\beta_{\mathrm{N}}$ limit, (c) poloidal beta $\beta_{\mathrm{p}}$, (d) loop voltage $V_{\text {loop }}$, (d) $D_{\alpha}$ emission from divertor revealing ELMy H-mode after $t=4.3 \mathrm{~s}$.

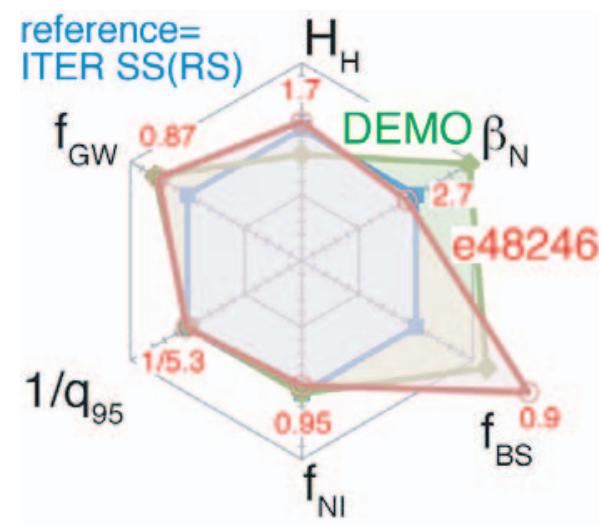

Fig. 4 Six figures of merit for JT-60U RS discharge in Figure 3, ITER SS (RS) and DEMO (SlimCS). Each figure of merit was normalized to that of ITER. Highly integrated performance was realized in wall-stabilized RS plasma in JT-60U.

the ITER SS scenario and are close to the DEMO regime (except $\beta_{\mathrm{N}}$ ).

The discharge in Figure 3 was terminated by the RWM at $\beta_{\mathrm{N}} \sim 2.7$ (close to ideal-wall beta limit of $\sim 2.9$ ) because of a decrease in the plasma rotation at the $q=3$ surface due to a change in the $q$ profile. Figure 5 provides the $q$ profile and toroidal rotation velocity profile at $t=5.4 \mathrm{~s}$ and $6.0 \mathrm{~s}$. The change in current profile resulted in the minimum $q$ $\left(q_{\text {min }}\right)$ decreasing and the $q=3$ surface that is resonant to

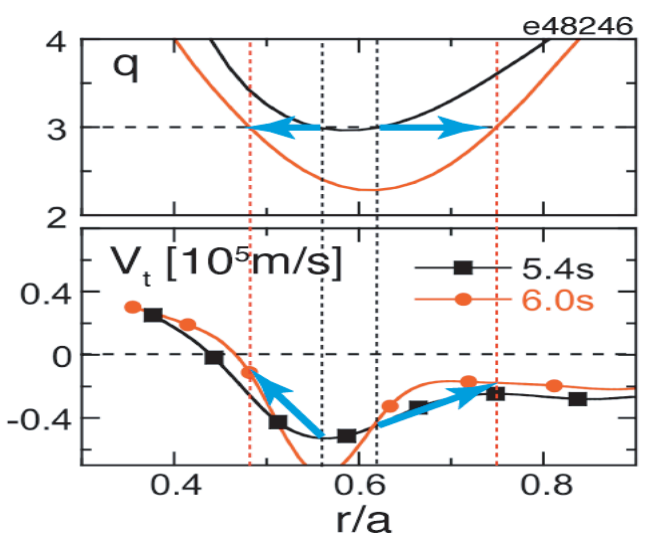

Fig. 5 Safety factor profiles and toroidal rotation profiles at $t=$ $5.4 \mathrm{~s}$ and $6.0 \mathrm{~s}$ in discharge shown in Figure 3. Toroidal rotation velocity at both $q=3$ surfaces mainly decreased due to the change in $q=3$ surface location.

$m / n=3 / 1$ mode changing. Mainly because of the change in $q=3$ surface location the toroidal rotation velocity at both $q=3$ surfaces decreased to the critical toroidal rotation velocity [9] $(\sim-20 \mathrm{~km} / \mathrm{s})$, finally resulting in the plasma being disrupted. More off-axis current drive is necessary in order to prevent the change in $q$ profile that takes place. The above result clearly reveals the realization of a steady current profile to be essential in the stable sustainment of that kind of high performance plasma.

\section{Realization of Fully Relaxed Cur- rent Profile in Full-CD High $f_{\mathrm{BS}}$ Plasma in JT-60U}

As described in the previous section the realization of a steady current profile is essential in the stable sustainment of a plasma developed in a steady state. In terms of the development of a plasma with a steady-state current profile for use in an AT operation scenario the key figures of merit of the above mentioned 6 figures of merit are $f_{\mathrm{BS}}$, $f_{\mathrm{NI}}$ and $1 / q_{95}$. The current profile to be developed must not depend on the inductive current profile, and hence $f_{\mathrm{NI}}$ must be unity. In an AT operation scenario as much as possible of the non-inductive current must be sustained by the bootstrap current to save on the cost of the external current drive, and therefore the $f_{\mathrm{BS}}$ must be sufficiently high, e.g. $f_{\mathrm{BS}}=0.46$ for an ITER SS (RS) scenario and $f_{\mathrm{BS}}$ $\sim 0.75$ for DEMO (SlimCS). Since the safety factor near edge $q_{95}$ affects the entire $q$ profile, and the characteristics of a plasma completely differ with different $q_{95}$ values, $q_{95}$ must also be an ITER/DEMO-relevant value $(\sim 5-6)$. The safety factor profile with a steady-state current profile should conform to the $q$ profile assumed in the AT operation scenarios presented in Figure 1. In order to satisfy the required key figures of merit, therefore, an operation scenario was developed utilizing the current drive capability of the JT-60U. 

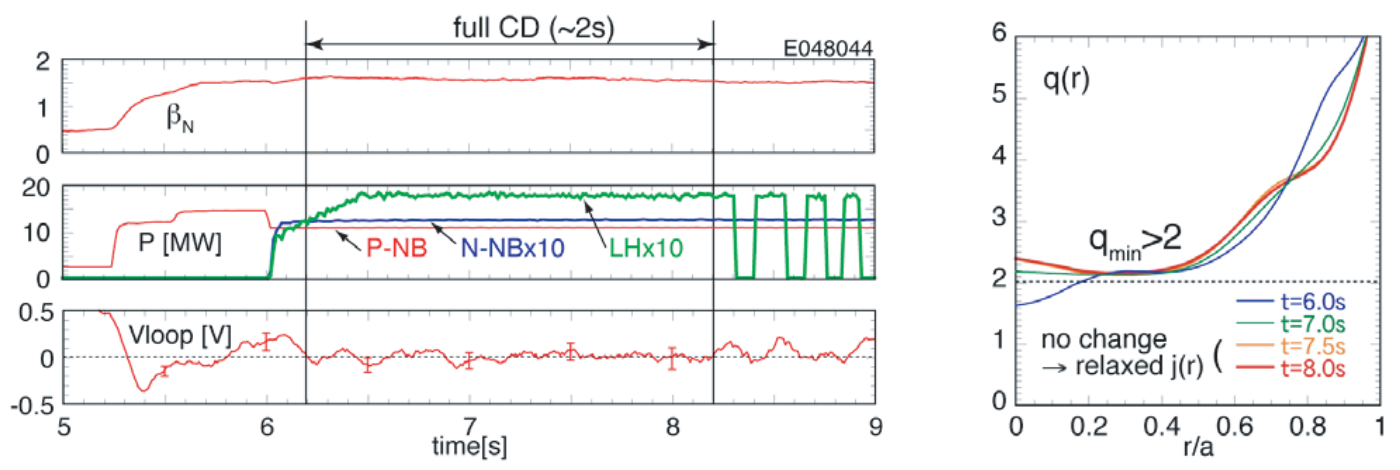

Fig. 6 Waveforms of discharge where fully relaxed current profile was realized in full-CD high $f_{\mathrm{BS}}=0.5$ plasma under ITER/DEMOrelevant $q_{95}=5.8$ : (a) normalized beta, (b) heating and current drive power of P-NB, N-NB and LH waves, (c) loop voltage. Temporal evolution of $q$ profile measured by MSE diagnostics in this discharge is provided in (d). The $q$ profiles at $t=7.5 \mathrm{~s}$ and $8.0 \mathrm{~s}$ are almost the same, revealing that the current profile had relaxed and a steady-state current profile was realized. Note that $q_{\min } \sim 2.1$ is maintained in a steady-state condition.

Figure 6 provides the waveforms of the developed discharge at $I_{\mathrm{p}}=0.8 \mathrm{MA}, B_{\mathrm{t}}=2.3 \mathrm{~T}$, and $q_{95}=5.8$ [10]. Preheating and a current drive using on-axis counter and offaxis co Positive-ion based Neutral Beams (P-NB) during $I_{\mathrm{p}}$ ramp-up was applied up to $t=5.2 \mathrm{~s}$ in order to prevent any decrease in $q$ near the center and maintain the $q$ profile as close as possible to the final steady-state $q$ profile that matches the AT $q$ profile. During the $I_{\mathrm{p}}$ flattop strong NB heating was applied in order to produce an Internal Transport Barrier (ITB) and H-mode edge and to raise the plasma pressure in creating more bootstrap current. After $t=6.0 \mathrm{~s}$ part of the P-NB heating power was replaced by current drive power using slightly off-axis Negative-ion based NB (N-NB, 1.2 MW at $320 \mathrm{keV}$ ) and off-axis LowerHybrid (LH, 1.8 MW) waves in order to reach the full-CD condition. As is seen in the decrease in loop voltage to $0 \mathrm{~V}$ the full-CD condition $\left(f_{\mathrm{NI}}=1\right)$ was realized during $t=6.2$ $8.2 \mathrm{~s}$. The full-CD condition was broken by notching of the LH power caused by arcing on the surface of the antenna. Since data on the poloidal magnetic flux within $\pm 0.1 \mathrm{~s}$ was used in calculating the $V_{\text {loop }}, V_{\text {loop }}$ started increasing about $0.1 \mathrm{~s}$ before the start of notching of LH power at $t \sim 8.3 \mathrm{~s}$.

The evolution of the $q$ profile over time measured through MSE diagnostics is plotted in Figure 6. During $t=6.0-7.5 \mathrm{~s}$ the q profile changed due to relaxation of the inductive electric field inside the plasma, however, the $q$ profiles at $t=7.5 \mathrm{~s}$ and $8.0 \mathrm{~s}$ are almost the same. This reveals the $q$ profile and current profile reached a steady state. Note that this steady-state $q$ profile has a $q_{\text {min }}$ of $\sim 2.1$ and hence no rational- $q$ surface that is resonant to the $m / n=1 / 1,3 / 2$, and $2 / 1$ modes, thus satisfying the necessary $q$ profile provided in the AT operation scenario in Figure 1 (weak magnetic shear regime). The $\beta_{\mathrm{N}} \sim 1.6$ achieved in this discharge was not limited by any MHD instability but instead by the current drive capability as the non-inductively driven current decreased at higher density.

In this plasma, the $f_{\mathrm{BS}}$ was calculated to be 0.5 using ACCOME code and measured temperature and density profiles. The fraction of the externally driven current with

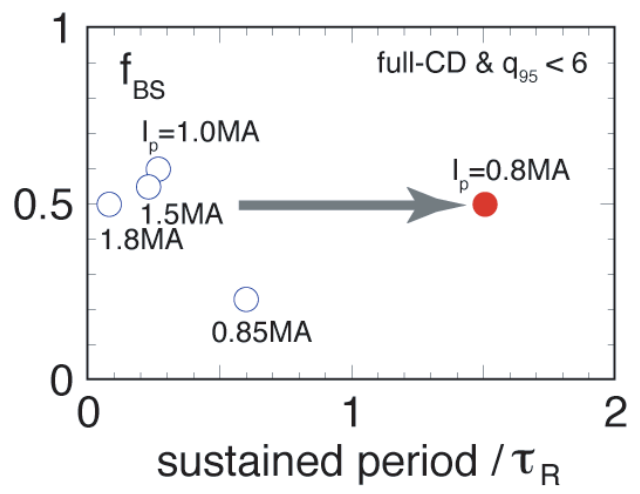

Fig. 7 The JT-60U operation regime was extended toward steady-state sustainment of full-CD plasma at high $f_{\mathrm{BS}}$. Filled circle is achieved in Figure 6. Open circles are previous achievements.

the LHCD and NBCD were 0.26 and 0.24 , respectively. A fully non-inductive discharge $f_{\mathrm{NI}}=1$ with a steady current profile was therefore realized at $f_{\mathrm{BS}}=0.5$ and an ITER/DEMO-relevant $q_{95} \sim 5.8$ in a weak magnetic-shear plasma regime at $q_{\mathrm{min}}=2.1$. Figure 7 provides the operation regime in $f_{\mathrm{BS}}$ and its sustained period under the full-CD condition in JT-60U, where discharges with $q_{95}<$ 6 were selected. The sustained period was normalized using the relaxation time of the current profile $\tau_{R}$ [4]. The operation regime was largely extended toward steady-state operation with a high $f_{\mathrm{BS}}$.

\section{AT Operation Scenarios Being Designed and to be Developed in JT-60SA for ITER and DEMO}

Total integration of the AT operation scenarios developed in JT-60U as well as extending the plasma performance need to be carried out for use with JT-60SA. With regard to the plasma performance (refer to Figure 4), although most of the figures of merit proved sufficiently high in JT-60U in view of ITER and DEMO, the $\beta_{\mathrm{N}}$ needs to be 
further extended to a higher value for DEMO. In order to realize the DEMO-equivalent $\beta_{\mathrm{N}}=4.3$ and to develop an optimum operation scenario JT-60SA is designed to posses the following key features in achieving a higher $\beta_{\mathrm{N}}$ than the no-wall $\beta_{\mathrm{N}}$ limit: (1) DEMO-relevant high shape parameter [11] $S \equiv q_{95} I_{\mathrm{p}} /\left(a B_{\mathrm{t}}\right) \sim 7$, where the $\beta_{\mathrm{N}}$ limit improves with increased $S$ [12], (2) stabilization through a closelyplaced conducting wall $(d / a \sim 1.2)$, (3) small ripple condition $(\sim 0.5-0.9 \%)$ for stabilization through a plasma rotation above critical rotation velocity $[9,13]$ with little braking effect on the toroidal rotation, (4) active feedback control of field-line deformation using in-vessel RWM control coils. The shape parameter and the ripple in the JT60U discharge shown in Figure 3 (e48246) were $\sim 2.3$ and $0.8 \%$ for $d / a \sim 1.3$, respectively. The ripple would become $\sim 1.3 \%$ for $d / a \sim 1.2$, if the plasma (e48246) was shifted outboard.

With regard to the steady-state operation and control of the current profile, JT-60SA is designed to possess the strong current drive capability of $10 \mathrm{MW} \mathrm{N-NB}$ at $500 \mathrm{keV}$ and $7 \mathrm{MW}$ of ECRF. An off-axis current driver is needed to realize and control the AT current profile revealed in Figure 1. With the JT-60SA, therefore, the beam line of the $\mathrm{N}-\mathrm{NB}$ is modified downward by $0.55 \mathrm{~m}$ when compared to the JT-60U. ECRF can be used not only to drive the plasma current but also to suppress or stabilize the NTM. The period of heating and current drive is $60-100 \mathrm{~s}$, which is sufficiently longer than the typical $\tau_{\mathrm{R}} \leq \sim 20 \mathrm{~s}$ [14].

Figure 8 provides an example of an AT operation regime for JT-60SA in terms of the 6 figures of merit and sustained period. Full-CD condition was investigated with ACCOME code, assuming DEMO-equivalent $H_{\mathrm{H} 98(\mathrm{y}, 2)}=$ 1.3 , fixed shape of temperature and density profiles, and the expected heating and current drive capability of $37 \mathrm{MW}$ (including $10 \mathrm{MW} \mathrm{N-NB}$ and $7 \mathrm{MW} \mathrm{ECRF)} \mathrm{with} I_{\mathrm{p}}=$ 2.3 MA, $B_{\mathrm{t}}=1.7 \mathrm{~T}$, and $q_{95}=5.7$. All the figures of merit of JT-60SA basically covered not only the ITER SS scenario but also nearly DEMO. A high $\beta_{\mathrm{N}}$ AT operation scenario sustained for a long period and reaching a relaxed current profile is the target of the JT-60SA in view of DEMO.

The end of this section concerns another important item to be developed in JT-60SA for DEMO. SlimCS DEMO [5] is an attractive concept in the realization of a compact reactor. A compact SlimCS design can be realized by reducing the volume (cross-section) of the Center Solenoid (CS, OH coil). The magnetic flux supplied by the $\mathrm{OH}$ coil $\Psi_{\mathrm{OH}}$ is proportional to the cross-section of the $\mathrm{OH}$ coil, and hence the $\Psi_{\mathrm{OH}}$ of SlimCS is insufficient to raise the $I_{\mathrm{p}}$ to the designed rated value of $16.7 \mathrm{MA}$. Figure 9 reveals the situation with the flux consumption of SlimCS. In a tokamak, the magnetic flux consumed to raise the $I_{\mathrm{p}}$ through induction from the $\mathrm{OH}$ coil is proportional to the destination $I_{\mathrm{p}}$. Typical tokamaks, e.g. JT-60U, JT60SA, and ITER, are designed to have sufficient magnetic flux to raise the $I_{\mathrm{p}}$ to their rated values through induction.
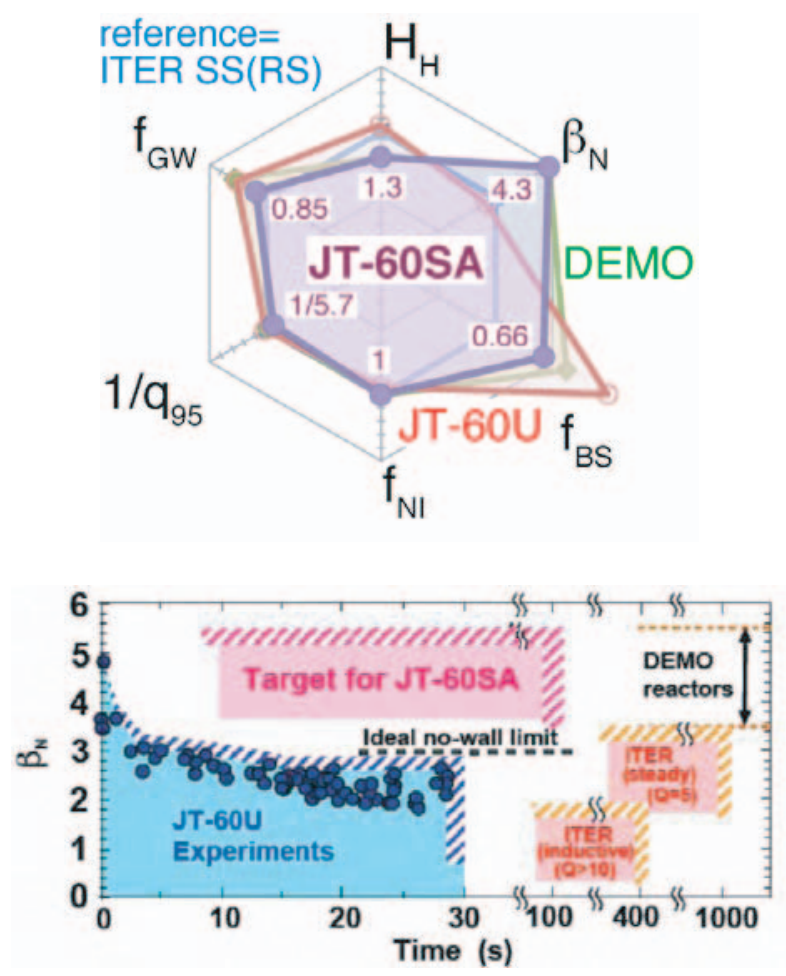

Fig. 8 Target operation regime of JT-60SA in (a) six figures of merit and (b) $\beta_{\mathrm{N}}$ as a function of sustained period. Achievements in JT-60U are plotted, and target regime of ITER and DEMO indicated in (b).

If the supply of the magnetic flux is insufficient another method of raising current is necessary. One possible example would be to raise the $I_{\mathrm{p}}$ non-inductively (route 1 in Figure 9) and another to recharge the consumed magnetic flux (route 2).

These $I_{\mathrm{p}}$ buildup scenarios were demonstrated using JT-60U [15]. Figure 10 reveals the discharge when $I_{\mathrm{p}}$ was raised non-inductively without any change in $\mathrm{OH}$ coil current (route 1 in Figure 9). NBCD with tangential P-NB and N-NB as well as ECCD were used in creating a noninductively driven current that included a bootstrap current larger than the $I_{\mathrm{p}}$. The plasma current increased from $215 \mathrm{kA}$ at $t \sim 3.8 \mathrm{~s}$ to $310 \mathrm{kA}$ at $t \sim 4.9 \mathrm{~s}$. Figure 11 reveals the discharge when the $\mathrm{OH}$ coil current was recharged non-inductively (route 2 in Figure 9) at $I_{\mathrm{p}}=0.6 \mathrm{MA}$ and $B_{\mathrm{t}}=4 \mathrm{~T}$ [15]. In this discharge a balanced injection of co/counter tangential NBs was employed to ensure that the non-inductive current was almost the same as the bootstrap current. During the $I_{\mathrm{p}}$ flattop recharging of the $\mathrm{OH}$ coil current under negative loop voltage was demonstrated (hatched region in Figure 11). The calculated $f_{\mathrm{BS}}$ was 1.05 in this discharge, even though the beam pressure component was conservatively neglected in calculating the bootstrap current. The two discharges in Figures 10 and 11 reveal that both the routes in Figure 9 can be used to raise the $I_{\mathrm{p}}$ non-inductively under an $f_{\mathrm{NI}}>1$ condition. Both of the discharges experienced collapse ( $t \sim 4.9 \mathrm{~s}$ in Figure 10) or disruption $(t \sim 5.3 \mathrm{~s}$ in Figure 11). Although the cause of 


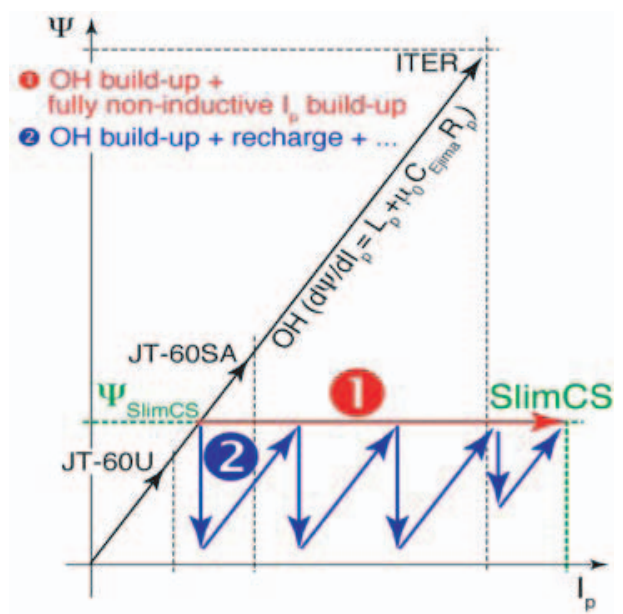

Fig. 9 Schematics of magnetic flux consumed as a function of $I_{\mathrm{p}}$. Magnetic flux is consumed in proportion to destination $I_{\mathrm{p}}$ when buildup is carried out by $\mathrm{OH}$ coil. Usual tokamaks have sufficient magnetic flux, whereas SlimCS does not. Non-inductive buildup of $I_{\mathrm{p}}$ (route 1) or recharging of $\mathrm{OH}$ coil (route 2) or some other way will be necessary with SlimCS.

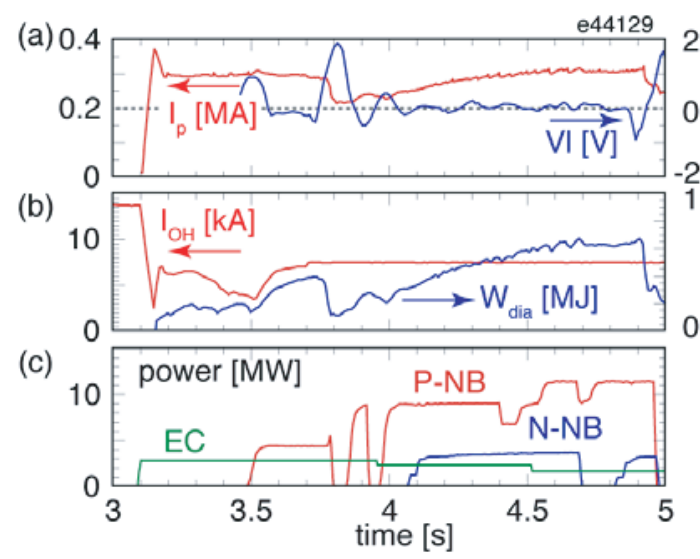

Fig. 10 Waveforms of non-inductive $I_{\mathrm{p}}$ buildup discharge in JT60U: (a) $I_{\mathrm{p}}$ and loop voltage $\mathrm{Vl}$, (b) $\mathrm{OH}$ coil current and diamagnetic stored energy $W_{\mathrm{dia}}$, (c) heating and current drive power by ECRF, P-NB and N-NB. Without any change of $\mathrm{OH}$ coil current, the plasma current increased at $f_{\mathrm{NI}}>1$.

the collapse or disruption in these discharges has not been recognized yet, one of the possible causes can be an MHD instability due to the change in current profile. Thus, control of the current profile during these $I_{\mathrm{p}}$ buildup scenarios would be attempted using N-NB in JT-60SA. Matching of these $I_{\mathrm{p}}$ buildup scenarios to the prelude phase of an integrated high performance plasma will also need to be investigated using JT-60SA and a total operation scenario developed using JT-60SA for DEMO.

\section{Summary}

The contribution of JT-60U and the foreseen JT-60SA to the development of an advanced tokamak operation sce-

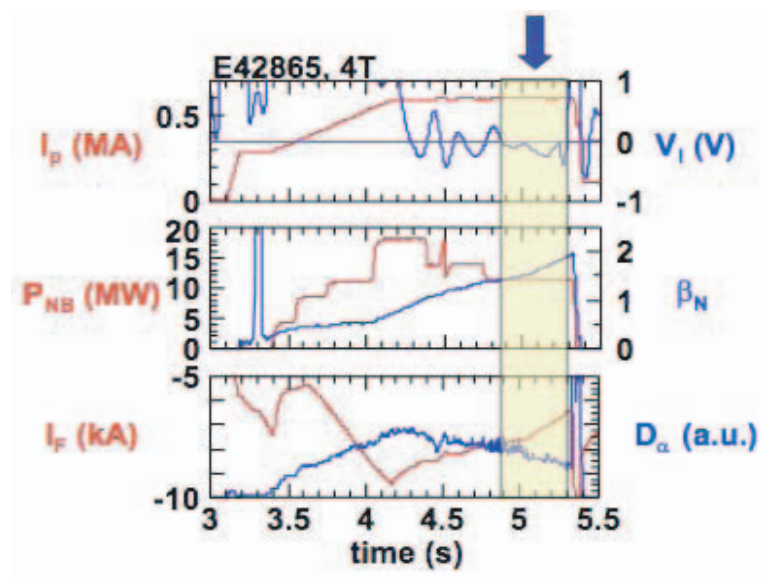

Fig. 11 Waveforms of discharge where recharging of $\mathrm{OH}$ coil was demonstrated: (a) $I_{\mathrm{p}}$ and loop voltage $V_{\mathrm{l}}$, (b) NB heating power $P_{\mathrm{NB}}$ and $\beta_{\mathrm{N}}$, (c) $\mathrm{OH}$ coil current $I_{\mathrm{F}}$, and $D_{\alpha}$ emission. Decrease in $\mathrm{OH}$ coil current corresponds to consumption of magnetic flux by plasma while increase corresponds to recharging of $\mathrm{OH}$ coil current by the plasma.

nario for ITER and DEMO has been presented. A high integrated performance plasma with a $\beta_{\mathrm{N}}$ of $\sim 2.7, H_{\mathrm{H} 98(\mathrm{y}, 2)}$ of $\sim 1.7, n_{\mathrm{e}} / n_{\mathrm{GW}}$ of $\sim 0.87$ and a very high $f_{\mathrm{BS}}$ of $\sim 90 \%$ at the DEMO/ITER relevant $q_{95}$ of $\sim 5.3$ was realized through stabilization by the closely placed conducting wall. A fully relaxed current profile was realized of a full-CD $\left(f_{\mathrm{NI}}=\right.$ 1) high $f_{\mathrm{BS}}=0.5$ plasma at $q_{95}=5.8$. The design of a JT-60SA operation scenario is steadily progressing toward steady-state advanced tokamak operation in ITER and DEMO. The fully non-inductive $I_{\mathrm{p}}$ buildup and noninductive recharging of the $\mathrm{OH}$ coil current (both $f_{\mathrm{NI}}>1$ ) demonstrated in JT-60U will contribute to the $I_{\mathrm{p}}$ buildup scenario development in JT-60SA for the SlimCS DEMO reactor concept. The scenario integration in JT-60SA will concern not only the core parameters presented here but also the overall tokamak system, including the divertor and wall e.g. heat load to divertor, recycling, etc.

[1] N. Oyama and the JT-60 Team, Nucl. Fusion 49, 104007 (2009).

[2] E. J. Doyle et al., Nucl. Fusion 47, S18 (2007).

[3] Plasma Performance Assessment (PPA), (August 2004).

[4] D. R. Mikkelsen, Phys. Fluids B 1, 333 (1989).

[5] K. Tobita et al., Nucl. Fusion 49, 075029 (2009).

[6] Y. Sakamoto et al., Nucl. Fusion 45, 574 (2005).

[7] H. Takenaga and the JT-60 Team, Nucl. Fusion 47, S563 (2007).

[8] Y. Sakamoto et al., Nucl. Fusion 49, 095017 (2009).

[9] M. Takechi et al., Phys. Rev. Lett. 98, 055002 (2007).

[10] T. Suzuki et al., Nucl. Fusion 49, 085003 (2009).

[11] E. A. Lazarus et al., Phys. Fluids B 4, 3644 (1992).

[12] M. R. Wade et al., Phys. Plasmas 8, 2208 (2001).

[13] H. Reimerdes et al., Phys. Rev. Lett. 98, 055001 (2007).

[14] T. Fujita et al., Nucl. Fusion 47, 1512 (2007).

[15] M. Ushigome et al., Nucl. Fusion 46, 207 (2006). 